\title{
Impacto de un programa personalizado de educación en pacientes diabéticos tipo 2
}

\section{Impact of a personalized education program in type 2 diabetic patients}

\author{
Adriana Medina, Édgar Javier Ellis, Diego Fernando Ocampo \\ - Bogotá, D.C. (Colombia)
}

\section{Resumen}

Los programas de educación en diabetes han logrado demostrar el beneficio en el control metabólico.

Objetivo: evaluar el impacto de un programa personalizado de educación para pacientes diabéticos tipo 2 en seguimiento a seis meses, en el Hospital de San José. Bogotá

Metodología: se condujo un estudio descriptivo retrospectivo a partir de los registros de pacientes adultos con diabetes tipo 2, que ingresaron al programa educativo entre enero 2011 y febrero 2012. Se realizaron intervenciones personalizadas y grupales con un equipo multidisciplinario liderado por médico endocrinólogo. Se compara el control metabólico al ingreso al programa y en el seguimiento al sexto mes según las metas propuestas por la ADA y ATP III.

Resultados: ingresaron al programa 115 pacientes, edad promedio de 61.1 años (DE 8.7); tiempo mediano de diagnóstico 10.5 años (RIQ 4-17.5), 43.9\% de los hombres y 54\% de las mujeres tenían más de 10 años de evolución. El 33.3\% de los hombres y 55.4\% mujeres presentaban obesidad. Los pacientes en metas de hemoglobina glicosilada A1c (HbA1c) en el seguimiento a seis meses, pasó de $10.4 \%$ al ingreso a $51 \%$, colesterol LDL de 41.9 a $47.1 \%$, HDL de 46.3 a $62.5 \%$ y triglicéridos de 25.6 a $50 \%$. Las pérdidas en el seguimiento fueron de $48.9 \%$. Se documentó asociación entre el tiempo de evolución de la enfermedad ( $\leq 5$ años) y las metas de HbA1c (p 0.005).

Conclusión: la experiencia en seguimiento a corto plazo de un programa educativo personalizado guiado por médico endocrinólogo y un equipo multidisciplinario favorece el control metabólico en pacientes diabéticos. (Acta Med Colomb 2014; 39: 258-263).

Palabras clave: diabetes mellitus, metabólico, educación.

\section{Abstract}

The diabetes education programs have succeeded in demonstrating benefit in metabolic control.

Objective: to evaluate the impact of a personalized education program for type 2 diabetic patients at 6 months follow-up, in the Hospital de San José, Bogotá.

Methodology: a retrospective descriptive study was conducted from records of adult patients with type 2 diabetes who were admitted to the educational program between January 2011 and February 2012. Personalized and group interventions conducted by a multidisciplinary team led by endocrinologist were realized. The metabolic control at program entry and at six months follow-up according to the goals set by the ADA and ATP III is compared.

Results: 115 patients were admitted to the program; average age 61.1 years (SD 8.7); median time from diagnosis 10.5 years (IQR 4-17.5), 43.9\% of men and 54\% of women were over 10 years of evolution. $33.3 \%$ of men and $55.4 \%$ women were obese. Patients in goals of glycosylated hemoglobin A1c (HbA1c) in the 6-month follow-up went from $10.4 \%$ on admission to 51\%, LDL cholesterol from $41.9 \%$ to $47.1 \%$, HDL from $46.3 \%$ to $62.5 \%$, and triglycerides from to $25.6 \% 50 \%$. Losses during follow-up were $48.9 \%$. Association between duration of disease $(\leq 5$ years) and goals of HbA1c (p $0.005)$ was documented.

Conclusion: the experience in short-term follow-up of a personalized educational program led by endocrinologist and a multidisciplinary team favors metabolic control in diabetic patients. (Acta Med Colomb 2014; 39: 258-263).

Keywords: diabetes mellitus, metabolic, education.

Dra. Adriana Medina Orjuela: Docente del Servicio de Endocrinología del Hospital San José, Instructor Asociado de la Fundación Universitaria de Ciencias de la Salud; Dr. Édgar Javier Ellis: Residente de Endocrinología Fundación Universitaria de Ciencias de la Salud, Hospital San José Dr. Diego Fernando Ocampo: Residente de Medicina Familiar Fundación Universitaria de Ciencias de la Salud, Hospital San José, Departamento de Endocrinología Hospital San José, Fundación Universitaria de Ciencias de la Salud. Bogotá, D.C. (Colombia). Correspondencia. Dr. Javier Elllis. Bogotá, D.C. (Colombia).

E-mail: javierellis78@hotmail.com Recibido: 1/XI/2012 Aceptado: 25/VIII/2014 


\section{Introducción}

La diabetes mellitus (DM) es el trastorno metabólico de origen multifactorial con mayor prevalencia a nivel mundial, convirtiéndose en una de las enfermedades crónicas más comunes con una alta morbilidad y mortalidad.(1)

Su prevalencia es de $2.8 \%$ y se cree que para 2030 será de $4.4 \%$, convirtiéndose en la quinta causa de muerte a nivel mundial. En países desarrollados como Estados Unidos esta enfermedad afecta a más de 20 millones de personas, generando gastos para su manejo y la de sus complicaciones que se estiman en 174 millones de dólares anuales y se prevé que en 2030 supere los 490 mil millones, convirtiéndose así en una enfermedad de alto costo. En el Reino Unido el gasto en salud para el manejo de este tipo de pacientes es de 4-5\% del presupuesto nacional (1). En Colombia se estima una prevalencia en mayores de 30 años de $7.5 \%$ (IC 95\% 5.1-9.8) que ajustada por sexo se obtienen datos para hombres, de $7.3 \%$ (IC: $95 \% 3.7-10.9$ ) y mujeres de $8.7 \%$ (IC: 95\% 5.2-12.3) (2).

Aproximadamente $10 \%$ de las personas con glicemia alterada en ayunas o intolerancia a los carbohidratos desarrollarán diabetes tipo $2(3,4)$, y sin intervención preventiva, alrededor de 50\% padecerá diabetes dentro de los 10 años (5). La manera más eficiente para manejar la diabetes mellitus tipo 2 y sus complicaciones es prevenir el desarrollo de la misma, así como lograr un buen control metabólico con el tratamiento instaurado (6-8).

El mayor riesgo de la diabetes mellitus está en relación con su pobre control y su asociación con enfermedades cardiovasculares micro y macrovasculares que conllevan a una alta morbilidad y mortalidad en este grupo de pacientes, incrementando los costos en el cuidado de la salud. Se han intentado introducir múltiples intervenciones desde políticas públicas, estrategias educativas y farmacológicas, con el fin de mejorar el control metabólico de estos pacientes y disminuir los costos en salud relacionados con el manejo de las complicaciones a corto y largo plazo (9-11).

Durante muchos años se han realizado múltiples intervenciones para intentar mejorar la adherencia a los manejos y mejorar control metabólico, estrategias como el llamado telefónico al paciente para recordarle las horas en la toma de medicamentos, el conteo de pastillas en cada visita a su médico, control con enfermería para la aplicación de insulina, el control con médicos de familia cada vez que el paciente lo requiera (12-15). Pero estas intervenciones no han contado con seguimiento por parte del médico endocrinólogo de forma permanente y grupos multidisciplinarios de forma permanente. Con cada una de estas intervenciones se consiguió una mejoría en metas hasta en 30\% según lo descrito en diferentes estudios (16-22).

El Hospital San José es una institución de cuarto nivel que atiende pacientes diabéticos en la consulta externa y hospitalización. Con frecuencia estos pacientes sólo son derivados a un nivel de mayor complejidad cuando ya ha progresado la enfermedad y presentan complicaciones. Este escenario supone retos mayores para un programa que debe educar y en ocasiones reentrenar pacientes en la cultura del autocuidado, no siempre para prevenir sino también para retardar la progresión de complicaciones cuando éstas ya se han documentado

Con este programa se intenta que el paciente tenga permanente contacto con un médico endocrinólogo y al mismo tiempo se hagan intervenciones para los cambios en el estilo de vida con la nutricionista y una educadora en diabetes que le pueda dedicar el tiempo necesario para que el paciente entienda mejor su enfermedad.

El objetivo es describir los resultados en control metabólico en el seguimiento a tres y seis meses de los pacientes con diabetes mellitus tipo 2 que ingresaron a un programa de educación personalizada en el Hospital San José de Bogotá.

\section{Metodología}

Se realizó un estudio descriptivo retrospectivo con revisión de historias clínicas de los pacientes con diagnóstico de diabetes mellitus tipo 2 del Hospital San José, en el periodo comprendido entre enero 2011 y febrero de 2012. Se incluyeron adultos mayores de 18 años con diagnóstico de diabetes tipo 2 por los criterios de la ADA. Se excluyeron pacientes con terapia de reemplazo renal.

Los pacientes que ingresaron al estudio fueron valorados por un especialista en endocrinología. Se les realizó un examen físico minucioso que incluyó peso, talla, cálculo del índice de masa corporal (IMC), perímetro abdominal, revisión de la realización de las glucometrías y en cada control se solicitó perfil lipídico completo, glicemia pre y posprandial y $\mathrm{HbA1c}$. Se documentaron los medicamentos recibidos por el paciente. Se realizó una consulta periódica cada tres meses o antes si el paciente requería un ajuste en el manejo. El mismo día del ingreso, el paciente fue valorado por la nutricionista encargada de la evaluación y explicación de los cambios en los hábitos alimentarios y posteriormente, la consulta de la educadora en diabetes, para valorar y promover cambios en el estilo de vida; la intervención educativa se realizó persona a persona y en presencia del familiar o cuidador.

Se analizan los datos disponibles de las variables de control metabólico al ingresar al programa, al tercero y sexto mes, como son: HbA1c, perfil de lípidos y los reportes de glucemias. Se reportan los cambios en las medidas antropométricas: IMC y perímetro abdominal.

El control metabólico se define como HbA1c menor o igual a 7.0\%. Las metas de lípidos se acogen a los criterios propuestos en el Adult Treatment Panel III guidelines (ATP III), colesterol LDL menor o igual a $100 \mathrm{mg} / \mathrm{dL}$, colesterol HDL mayor o igual a $40 \mathrm{mg} / \mathrm{dL}$ y triglicéridos menores o iguales a $150 \mathrm{mg} / \mathrm{dL}$.

Para el análisis de los datos se utilizó el programa Stata $12.0^{\circledR}$. Mediante estadística descriptiva se resumen las variables categóricas en frecuencias absolutas y porcentajes y las variables cuantitativas con medidas de tendencia central y dispersión. Se reporta la proporción de pacientes 
que cumplen metas terapéuticas al ingreso y en el control a tres y seis meses de seguimiento, según la distribución por sexo y el tiempo de evolución de la enfermedad. La prueba t de Student para muestras pareadas se utiliza para presentar diferencias en los promedios de la HbA1c y lípidos antes del ingreso al programa, con el control a los seis meses se definió como el nivel de significancia un valor $\mathrm{p}<0.05$.

El protocolo fue aprobado por el Comité de Investigaciones de la Facultad de Medicina de la Fundación Universitaria de Ciencias de la Salud y el comité de ética en investigación con seres humanos, según la Resolución 8430 del Ministerio de Salud se considera una investigación sin riesgo.

\section{Resultados}

Ingresaron al programa 115 pacientes con diagnóstico de diabetes mellitus tipo 2. Las características de la población se presentan en la Tabla 1. Los datos se registraron en la primera visita al servicio de endocinología, por esta razón hay 19 pacientes que al ingreso no tienen el dato de $\mathrm{HbA1c}$, aún habiéndola solicitado traer en los siguientes días a esta visita. La edad promedio al ingreso en el programa fue de
62.2 (DE 8.9) años para los hombres y 60.2 (DE 8.6) años para las mujeres. El tiempo mediano de diagnóstico de la enfermedad fue 10.5 años (RIQ 4- 17.5) con ausencia de datos para 11 pacientes, se documentó que cuatro (3.9\%) tenían menos de un año de diagnóstico, entre 1 y 4.9 años, 33 pacientes $(31.7 \%)$; entre cinco y diez años, 15 pacientes (14.4\%) y 52 pacientes (50\%) tenían más de diez años de evolución de la enfermedad.

Se revisaron los índices de masa corporal con el que ingresaron los pacientes. Cerca de la mitad presentaban obesidad (46\%), se encontraban en sobrepeso 46 pacientes (40.7\%) y sólo 15 pacientes tenían normopeso (13.3\%). Entre las mujeres fue mayor la presencia de obesidad $55.4 \%$ respecto a $33.3 \%$ en los hombres (p 0.04). La mediana del perímetro abdominal encontrado al inicio del estudio para hombres fue de $110 \mathrm{~cm}$ (RIQ 104-114) y para mujeres 105 cm (RIQ 99-113).

Al analizar el estado metabólico antes del ingreso al programa educativo se identificaron 10 pacientes (10.4\%) en metas de $\mathrm{Hb} \mathrm{A} 1 \mathrm{c}, 37$ pacientes $(46.3 \%)$ en metas de HDL, 26 pacientes (41.9\%) en metas de LDL y 21 pacientes

Tabla 1. Características de la población al ingreso al programa.

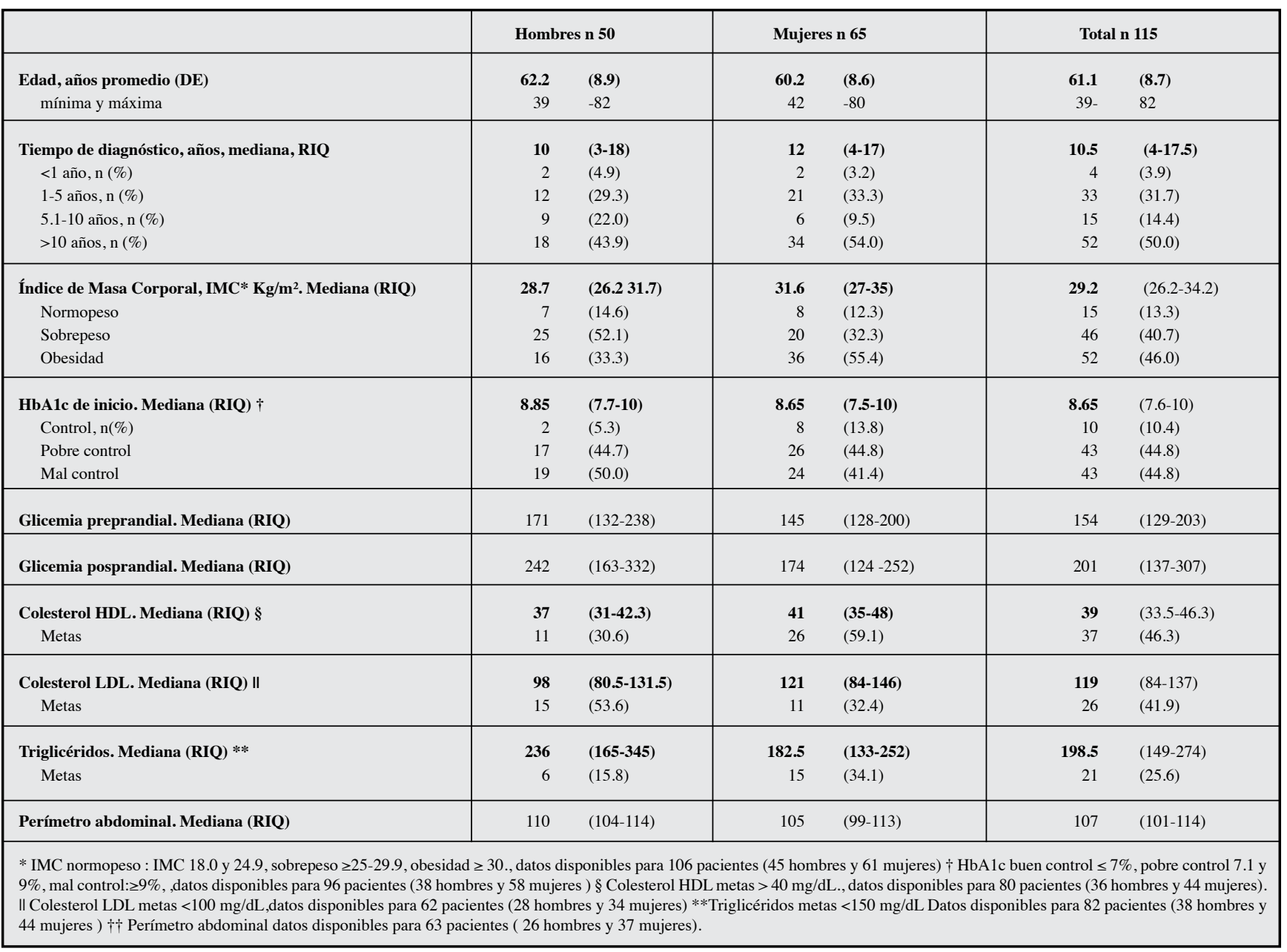


(25.6\%) en metas terapéuticas de triglicéridos. La mediana de glicemias preprandiales para hombres fue de 171 (RIQ 132-238), para mujeres $145 \mathrm{mg} / \mathrm{dL}$ (RIQ 128-200).

Los pacientes que lograron metas a los tres y a los seis meses de seguimiento en el programa se muestran en la Tabla 2. A los tres meses de seguimiento tuvieron control de la $\mathrm{HbA} 1 \mathrm{C} 20 / 63$ pacientes $(31.8 \%)$. Alcanzaron metas de HDL, 23/51 pacientes (45.1\%), LDL, $16 / 40$ pacientes (40\%) y triglicéridos $20 / 44$ pacientes $(45.4 \%)$. En el seguimiento a los seis meses lograron control de HbA1c 25/49 pacientes (51.0\%), HDL, 30/48 pacientes (62.5\%), LDL 16/34 pacientes $(47.1 \%)$ y triglicéridos $20 / 40$ pacientes $(50 \%)$. Los cambios en los niveles de HbA1c, LDL, HDL y triglicéridos se muestran en las Figuras 1 a 4 .
Al finalizar el seguimiento a los seis meses las pérdidas para cada una de las variables fue para $\mathrm{HbA1c} 48.9 \%$, para HDL $33.3 \%$, para LDL $45.2 \%$ y para triglicéridos $51.2 \%$.

El análisis según el tiempo de evolución de la diabetes, mostró diferencias estadísticamente significativas en los niveles de HbA1c al ingreso al programa en pacientes con menos de cinco años y aquellos con más de cinco años de diagnóstico de la enfermedad [8.4\% (DE 1.8\%) vs $9.3 \%$ (DE 1.9\%); p 0.043], diferencias que se mantienen en el seguimiento a seis meses $(6.5 \%$ (DE $0.68 \%$ ) vs $7.4 \%$ (DE $1.1 \%) ; \mathrm{p} 0.0054)$. Al ingreso al programa se encontraban en metas de HbA1c, el 17.2\% (5/29) de los pacientes con menos de cinco años de diagnóstico, y en quienes tenían más de cinco años de evolución, el 5.2\% (3/58) cumplía metas. En

Tabla 2. Cumplimiento de metas terapéuticas HbAlc y perfil de lípidos, según la distribución por sexo, en seguimiento a tres y seis meses.

\begin{tabular}{|c|c|c|c|c|c|c|}
\hline HbA1C, n (\%) & \multicolumn{2}{|c|}{ Inicio } & \multicolumn{2}{|c|}{3 meses } & \multicolumn{2}{|c|}{6 meses } \\
\hline $\begin{array}{l}\text { Hombres } \\
\text { Mujeres } \\
\text { Total }\end{array}$ & $\begin{array}{r}2 / 38 \\
8 / 58 \\
10 / 96\end{array}$ & $\begin{array}{l}(5.3) \\
(13.8) \\
(10.4)\end{array}$ & $\begin{array}{r}7 / 27 \\
13 / 36 \\
20 / 63\end{array}$ & $\begin{array}{l}(25.9) \\
(36.1) \\
(31.8)\end{array}$ & $\begin{array}{r}6 / 15 \\
19 / 34 \\
25 / 49\end{array}$ & $\begin{array}{l}(40.0) \\
(55.9) \\
(51.0)\end{array}$ \\
\hline $\begin{array}{l}\text { Colesterol HDL, n (\%) } \\
\text { Hombres } \\
\text { Mujeres } \\
\text { Total }\end{array}$ & $\begin{array}{l}11 / 36 \\
26 / 44 \\
37 / 80\end{array}$ & $\begin{array}{l}(30.1) \\
(59.1) \\
(46.3)\end{array}$ & $\begin{array}{r}8 / 24 \\
15 / 27 \\
23 / 51\end{array}$ & $\begin{array}{l}(33.3) \\
(55.6) \\
(45.1)\end{array}$ & $\begin{array}{l}10 / 18 \\
20 / 30 \\
30 / 48\end{array}$ & $\begin{array}{l}(55.6) \\
(66.7) \\
(62.5)\end{array}$ \\
\hline $\begin{array}{l}\text { Colesterol LDL, } \mathbf{n}(\%) \\
\text { Hombres } \\
\text { Mujeres } \\
\text { Total }\end{array}$ & $\begin{array}{l}15 / 28 \\
11 / 34 \\
26 / 62\end{array}$ & $\begin{array}{l}(53.6) \\
(32.4) \\
(41.9)\end{array}$ & $\begin{array}{r}8 / 20 \\
8 / 20 \\
16 / 40\end{array}$ & $\begin{array}{l}(40.0) \\
(40.0) \\
(40.0)\end{array}$ & $\begin{array}{r}5 / 12 \\
11 / 22 \\
16 / 34\end{array}$ & $\begin{array}{l}(41.7) \\
(50.0) \\
(47.1)\end{array}$ \\
\hline $\begin{array}{l}\text { Triglicéridos, n (\%) } \\
\text { Hombres } \\
\text { Mujeres } \\
\text { Total }\end{array}$ & $\begin{array}{r}6 / 38 \\
15 / 44 \\
21 / 82\end{array}$ & $\begin{array}{l}(15.8) \\
(34.1) \\
(25.6)\end{array}$ & $\begin{array}{r}6 / 20 \\
14 / 25 \\
20 / 44\end{array}$ & $\begin{array}{l}(30.0) \\
(56.0) \\
(45.4)\end{array}$ & $\begin{array}{r}7 / 14 \\
13 / 26 \\
20 / 40\end{array}$ & $\begin{array}{l}(50.0) \\
(50.0) \\
(50.0)\end{array}$ \\
\hline $\begin{array}{l}\text { Perímetro abdominal. cm, mediana (RIQ) } \\
\text { Hombres } \\
\text { Mujeres }\end{array}$ & $\begin{array}{l}107 \\
110 \\
105\end{array}$ & $\begin{array}{l}(101-114) \\
(104-114) \\
(99-113)\end{array}$ & $\begin{array}{r}103 \\
108 \\
99,5\end{array}$ & $\begin{array}{l}(96-110) \\
(102-110) \\
(96-110)\end{array}$ & $\begin{array}{l}102 \\
103 \\
102\end{array}$ & $\begin{array}{l}(100-109) \\
(100-107.5) \\
(97-110)\end{array}$ \\
\hline
\end{tabular}

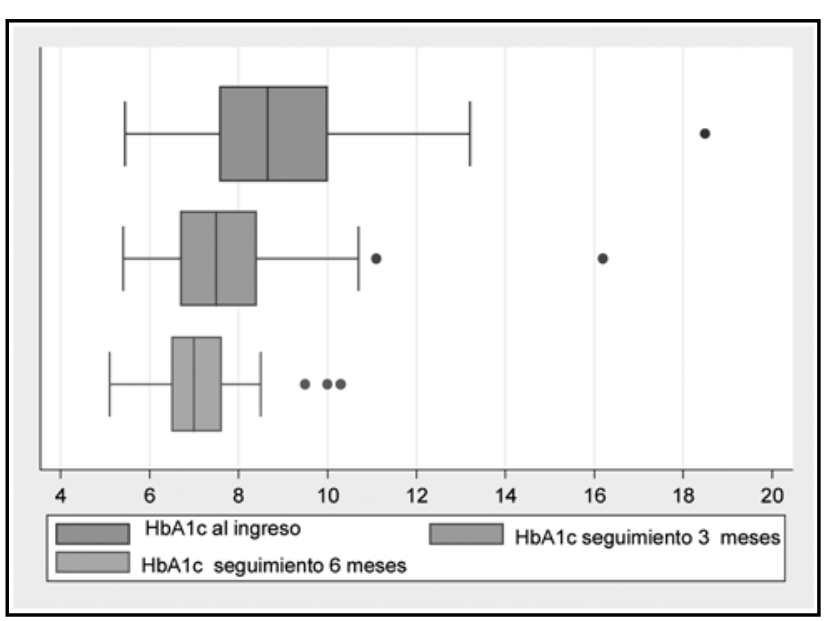

Figura 1. Cambio en niveles de HbAlc (\%).

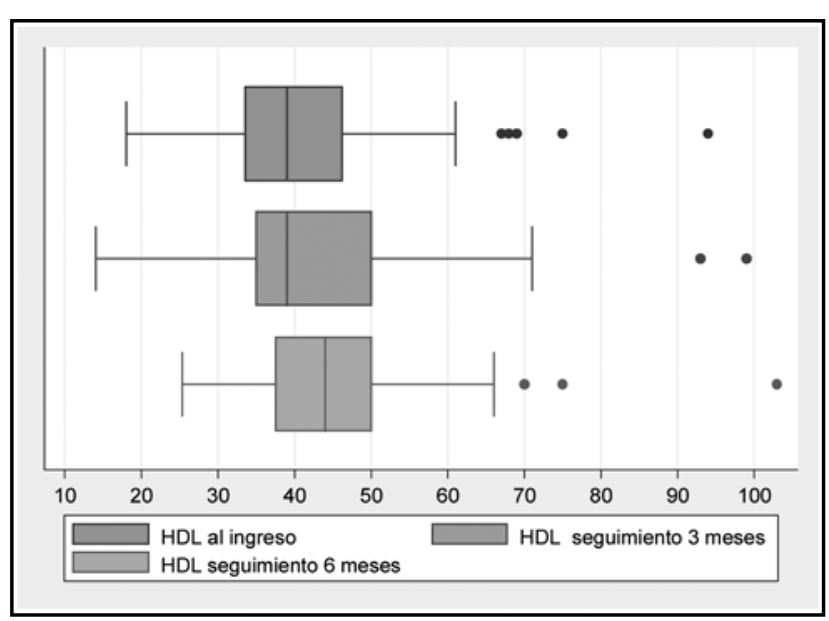

Figura 2. Cambios en niveles de colesterol $H D L(m g / d L)$. 


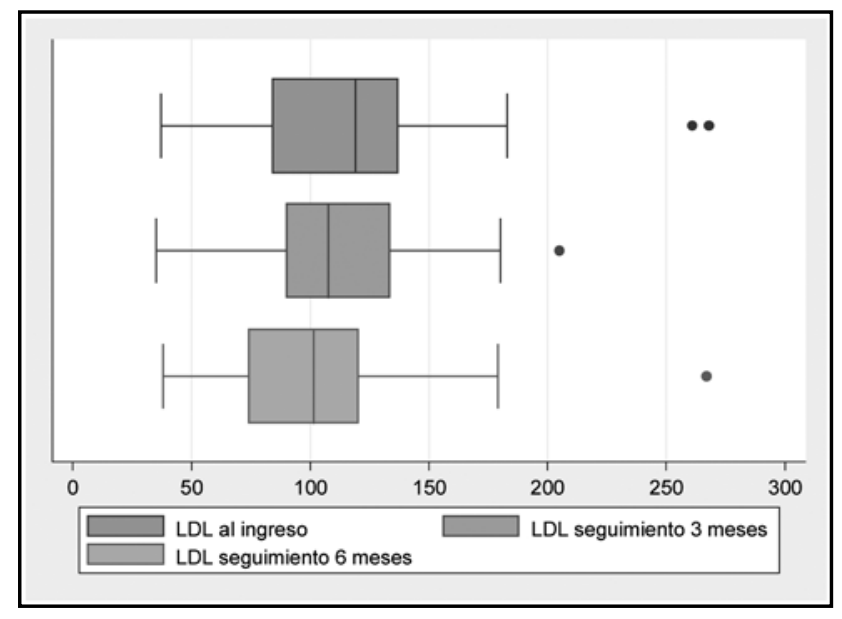

Figura 3. Cambios en niveles de colesterol $L D L(\mathrm{mg} / \mathrm{dL})$.

el seguimiento a seis meses alcanzaron metas de $\mathrm{HbAlc}$ el $80 \%$ de los pacientes (12/15) con menos de cinco años de evolución de la diabetes y el 40.6\% (13/32) de los pacientes con más de cinco años de evolución de la enfermedad.

Entre los 46 pacientes que cumplieron el seguimiento a seis meses y tenían datos de $\mathrm{HbA} 1 \mathrm{c}$, todos presentaron reducción estadísticamente significativa del nivel de HbAlc, respecto al basal $(\mathrm{p}<0.001)$, la mediana de la reducción fue $1.2 \%$ (RIQ $0.7 \%-2 \%$ ). El cambio promedio en niveles lípidos no mostró resultados estadísticamente significativos LDL (p 0.123 ), HDL ( $\mathrm{p} 0.459$ ), triglicéridos ( $\mathrm{p} 0.05$ ). Aunque no fue el objetivo primario de este estudio, se realizó un análisis exploratorio con este subgrupo de pacientes y se identificó que el tener menos de cinco años de evolución de la enfermedad se asocia con cumplimiento de metas de HbA1c (p 0.005).

\section{Discusión}

En este estudio observacional de 115 pacientes con diabetes tipo 2 que ingresaron por primera vez a un programa de educación en diabetes, se pretende mostrar las ventajas que tiene sobre ellos, no sólo el hecho de asistir a una cita médica endocrinológica, sino además de aprender a cambiar estilos de vida al recibir mayor información con respecto a su patología y la importancia de prevenir complicaciones, mediante un mejor control metabólico.

El promedio de edad de los pacientes que ingresaron al programa se encuentra entre los 62.2 años para los hombres y 60.2 años para las mujeres.

En el estudio del Dr. Aschner (2) sobre la epidemiología de la diabetes en Colombia del año 2010, se encontró una prevalencia de $7.3 \%$ de diabetes en hombres mayores de 30 años y de $7.4 \%$ en mujeres, similar a los datos encontrados en el estudio CARMELA con una mayor prevalencia en mujeres y con una población hasta de 64 años. En Colombia la prevalencia de diabetes mellitus tipo 2 oscila entre 4 y $8 \%$, encontrándose entre las primeras cinco causas de muerte, lo cual concede toda la importancia a su prevención $\mathrm{y}$ adecuado tratamiento.

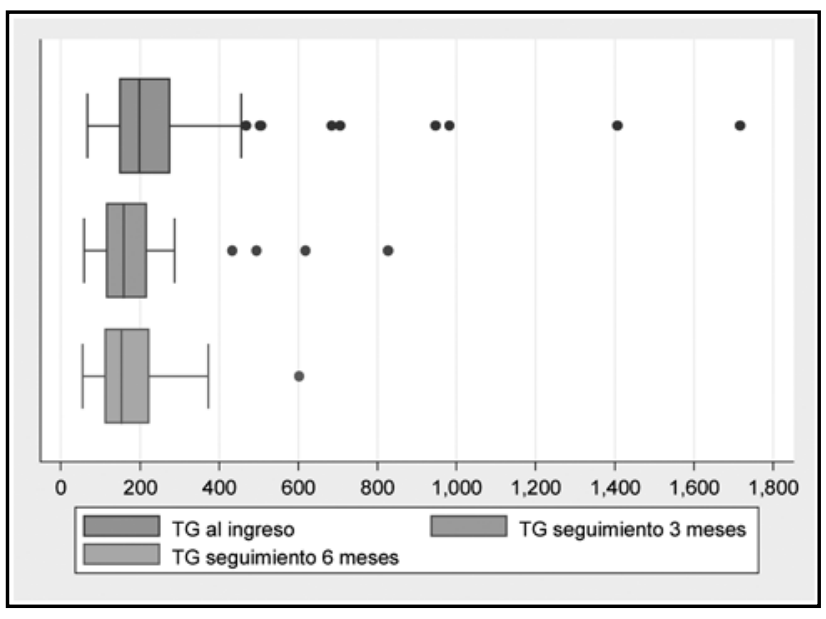

Figura 4. Cambios en niveles de triglicéridos $(\mathrm{mg} / \mathrm{dL})$.

Para el momento en que el paciente ingresa al programa, el tiempo que llevaba de diagnóstico de la enfermedad, fue tanto para hombres como mujeres en su mayoría, superior a cinco años, tiempo durante el cual el paciente ha podido desarrollar complicaciones secundarias a la diabetes, lo cual puede estar en relación con la ausencia de cambios en el estilo de vida, la irregularidad en la toma de la medicación o la falta de control metabólico a pesar de la toma de la misma. Con este programa se intenta enrolar pacientes para seguimiento de seis meses con el fin de disminuir estas complicaciones al lograr un cambio en el estilo de vida mediante la educación personalizada que le permita al paciente entender su enfermedad, además de poder ser visto de forma oportuna, si no se ha logrado un adecuado control metabólico.

Durante el seguimiento que se les realizó a los pacientes durante este corto periodo de seis meses, con visitas en intervalos de cada tres meses, donde recibieron consulta médica endocrinológica y al mismo tiempo se intervenía por la educadora en diabetes y nutricionista, se logró disminuir en los pacientes que continuaron durante el programa, los parámetros de control metabólico como HbA1c, colesterol total, colesterol LDL, triglicéridos y colesterol HDL, que están en relación con el desarrollo de complicaciones macrovasculares, lo que podría representar en el futuro menor morbilidad, mortalidad y mejor calidad de vida para este tipo de pacientes.

Con respecto a las metas metabólicas los pacientes que lograron $\mathrm{HbA} 1 \mathrm{C}$ menor de $7 \%$ pasó de $10.4 \%$ al inicio del programa a $51.0 \%$ a los seis meses. Los pacientes que ingresaron a nuestro estudio mejoraron todos los parámetros del perfil lipídico al final del seguimiento, logrando metas en los niveles de colesterol LDL en $47.1 \%$, de triglicéridos en $50 \%$ y de HDL en $58.3 \%$ a los seis meses de intervención. En contraste, el Dr. Deakin en su metaanálisis en 2009 publicado por Cochrane no encontró diferencias estadísticas al valorar los triglicéridos a los tres, seis y 12 meses de seguimiento. Desafortunadamente, en este estudio, no hay datos de colesterol total, LDL y HDL (23). 
La limitación de nuestro estudio fue la pérdida de la población que se tuvo durante el seguimiento del programa que puede estar en relación con la falta de interés del paciente por su enfermedad, por los cambios que constantemente presentan las EPS (empresas promotoras de salud) en su contratación institucional, por el tiempo de seguimiento de cada tres meses o la falta de demanda inducida que se pudo hacer a los pacientes dentro del cual puede estar el seguimiento telefónico.

Según los datos reportados por el Dr. Vermeire en su revisión en Cochrane en 2009, se encontró que al realizar intervenciones como programas de seguimiento de diabetes, en este estudio sólo con enfermería, se presentó una disminución en $\mathrm{HbA} 1 \mathrm{c}$ de $0.4 \%$, pero no en otras variables como perfil lipídico, concluyendo, además, que la mayoría de los estudios sobre adherencia a programas de diabetes están muy limitados por la pérdida de datos o datos no confiables y bajo número de participantes (24), la Dra. Duke en su revisión Cochrane demuestra pérdidas variables en las poblaciones unas tan altas como de $64 \%$, y otras tan bajas como de $1 \%$ con este tipo de intervenciones, trayendo dificultades para un seguimiento estricto (25).

En nuestro estudio, la pérdida de datos a los seis meses para cada una de las variables fue para HBA1c $48.9 \%$, para HDL $33.3 \%$, para LDL $45.2 \%$ y para triglicéridos $51.2 \%$.

Se encontró de manera adicional, que la HbA1c era más baja a menos tiempo de diagnóstico de la diabetes ( $<5$ años), lo cual indica que el tiempo de evolución estaría relacionado con mal control glicémico, así como lo demuestran estudios como el UKPDS y Longo-Mbenza en un estudio sobre retinopatía diabética $(26,27)$.

\section{Conclusiones}

Un programa para diabéticos multidisciplinario bien estructurado y guiado por médicos endocrinólogos, mejora las metas metabólicas de los pacientes con diabetes tipo 2.A largo plazo esto podría representar un impacto en la calidad de vida de los pacientes con menores índices de morbilidad y mortalidad y al mismo tiempo ese impacto se verá reflejado en el sistema de salud del país, al disminuir las complicaciones que esta enfermedad conlleva y los gastos en salud.

Las metas en el control metabólico en los pacientes diabéticos no son difíciles de conseguir, si el paciente está interesado en modificar sus hábitos de vida, y tiene a su lado un equipo médico multidisciplinario que lo guíe y esté dispuesto a atenderlo cuando lo necesite.

Se deben buscar estrategias institucionales y gubernamentales para lograr que un grupo de pacientes que ingresa a un programa de educación en diabetes tenga continuidad y de esta manera no se pierda de los beneficios que éste trae en su control metabólico.

Sólo el seguimiento a largo plazo permite evaluar el impacto de estas intervenciones, pero el mantener al paciente en metas terapéuticas es el camino para la reducción de la morbimortalidad por diabetes mellitus.

\section{Referencias}

1 International Diabetes Federation. Diabetes Atlas. International Diabetes Federation 2010.

2 Aschner M. Epidemiología de la diabetes en Colombia. Avances en diabetología 2010; 26(2): 95-100.

3 Federación Diabetologica Colombiana. Periodico. Periodico 2[3], 1. 2011 2-8-2011.

4 Cheng D. Prevalence, predisposition and prevention of type II diabetes. Nutr Metab(Lond) 2005; 2: 29.

5 Lindstrom J, Ilanne-Parikka P, Peltonen M, Aunola S, Eriksson JG, Hemio $\mathbf{K}$, et al. Sustained reduction in the incidence of type 2 diabetes by lifestyle intervention: follow-up of the Finnish Diabetes Prevention Study. Lancet 2006; 368(9548): 1673-9.

6 Schwarz PE, Bornstein SR. Pre-diabetes and metabolic syndrome in Germans Horm Metab Res 2006; 38(5): 359

7 Cano-De La Cuerda R, Aguila-Maturana AM, Miangolarra-Page JC. [Effectiveness of physical exercise programs in patients with diabetes mellitus]. Med Clin (Barc) 2009; 132(5): 188-94.

8 Baker MK, Simpson K, Lloyd B, Bauman AE, Singh MA. Behavioral strategies in diabetes prevention programs: a systematic review of randomized controlled trials. Diabetes Res Clin Pract 2011; 91(1): 1-12.

9 Lorig K, Ritter PL, Laurent DD, Plant K, Green M, Jernigan VB, et al. Online diabetes self-management program: a randomized study. Diabetes Care 2010; 33(6): $1275-81$.

10 Naik AD, Palmer N, Petersen NJ, Street RL, Jr., Rao R, Suarez-Almazor M, et al. Comparative effectiveness of goal setting in diabetes mellitus group clinics: randomized clinical trial. Arch Intern Med 2011; 171(5): 453-9.

11 Rygg LO, Rise MB, Gronning K, Steinsbekk A. Efficacy of ongoing group based diabetes self-management education for patients with type 2 diabetes mellitus. A randomised controlled trial. Patient Educ Couns 2011.

12 Espinet LM, Osmick MJ, Ahmed T, Villagra VG. A cohort study of the impact of a national disease management program on HEDIS diabetes outcomes. Dis Manag 2005; 8(2): 86-92.

13 Mangione CM, Gerzoff RB, Williamson DF, Steers WN, Kerr EA, Brown AF, et al. The association between quality of care and the intensity of diabetes disease management programs. Ann Intern Med 2006; 145(2):107-16.

14 Malathy R, Narmadha M, Ramesh S,Alvin JM, Dinesh BN. Effect of a diabetes counseling programme on knowledge, attitude and practice among diabetic patients in Erode district of South India. J Young Pharm 2011; 3(1): 65-72.

15 Fortmann AL, Gallo LC, Philis-Tsimikas A. Glycemic control among Latinos with type 2 diabetes: the role of social-environmental support resources. Health Psychol 2011; 30(3): 251-8.

16 Magee M, Bowling A, Copeland J, Fokar A, Pasquale P, Youssef G. The ABCs of diabetes: diabetes self-management education program for African Americans affects A1C, lipid-lowering agent prescriptions, and emergency department visits. Diabetes Educ 2011; 37(1): 95-103.

17 Mazze RS, Bergenstal R, Ginsberg B. Intensified diabetes management: lessons from the diabetes control and complications trial. Int J Clin Pharmacol Ther 1995; 33(1): 43-51.

18 Renders CM, Valk GD, Griffin S, Wagner EH, Eijk JT, Assendelft WJ. Interventions to improve the management of diabetes mellitus in primary care, outpatient and community settings. Cochrane Database Syst Rev 2001; (1): CD001481.

19 Norris SL, Engelgau MM, Narayan KM. Effectiveness of self-management training in type 2 diabetes: a systematic review of randomized controlled trials. Diabetes Care 2001; 24(3): 561-87

20 Yu GC, Beresford R. Implementation of a chronic illness model for diabetes care in a family medicine residency program. J Gen Intern Med 2010; 25 Suppl 4: S615-9.

21 Tejada T, Pastor D, Gutiérrez E. Efectividad de un programa educativo en el control del enfermo con diabetes. Invest Educ Enferm 2006; 24(2): 48-53.

22 Gallegos C, Bañuelos Y. Conductas protectoras de salud en adultos con diabetes mellitus tipo II. Invest Educ Enferm 2004; 22(2): 40-9.

23 Trudi A, Deakin C. Group based training for self-management strategies in people with type 2 diabetes mellitus. The Cochrane Collaboration Published by JohnWiley \& Sons 2012; (2): 1-83.

24 Etienne I, Vermeire J, et al. Interventions for improving adherence to treatment recommendations in people with type 2 diabetes mellitus. The Cochrane Collaboration Published by JohnWiley \& Sons 2009; (1): 1-44.

25 Sally-Anne $S$ Duke $S$. Individual patient education for people with type 2 diabetes mellitus. The Cochrane Collaboration Published by John Wiley \& Sons 2009; (1) $1-45$.

26 Clarke P, et al. A model to estimate the lifetime health outcome of patients with Type 2 diabetes: the United Kingdom Prospective Diabetes Study (UKPDS) Outcomes Model (UKPDS no. 68). Diabetologia 2008; 47(10): 1747-59.

27 Longo-Mbenza B, et al. Risk factors of poor control of HBA1c and diabetic retinopathy: Paradox with insulin therapy and high values of HDL in African diabetic patients. Int J Diabetes \& Metabolism 2008; (16): 69-78. 